\title{
SIMULATION STUDY OF COHERENT HIGH HARMONIC* GENERATION ON NIJI-VI STORAGE RING
}

\author{
Hongliang $\mathrm{Xu}^{\dagger}$, Jinying Liu, Duohui He, NSRL, USTC, P. R. China \\ Kawakatsu Yamada, Norihiro Sei, Tetsuo Yamazaki, ETL, Japan
}

\begin{abstract}
In this paper, three kinds of experimental designs to generate coherent high harmonic wave were mainly discussed in NIJI-VI storage ring, and the feasibility of these methods were analyzed to find the optimal experimental design. Especially when the second undulator magnetic field period keep constant and only its gap was changed for its resonant wavelength equal to third harmonic wavelength of the first undulator, the coherent emitting intensity of optical klystron can be improved under some conditions.
\end{abstract}

\section{Introduction}

In theoretical concept, FEL is an extremely adaptable light source which can produce high power coherent radiation virtually over entire electromagnetic spectrum; but now actually FEL only reaches UV or more better for deep $\mathrm{UV}^{[1,2]}$ which wavelength is $208 \mathrm{~nm}$, and efforts to shorten the FEL wavelength down to deep UV range mainly by using storage rings, in which high energy beams with good quality can be obtained; in this case, it is quite difficult to achieve more shorter, even X-ray FEL, because of the decrease of the gain and the lackness high-reflectivity mirrors. Thus coherent harmonic generation $(\mathrm{CHG})$ technique $\mathrm{e}^{[3,4,5]}$ was proposed and some good results are obtained ${ }^{[6,7]}$, and other research groups are being ready for $\mathrm{CHG}$ investigation, such as NSRL in China. On NIJI-VI storage ring in ETL, CHG experimental investigation program is being carried out ${ }^{[1]}$, and attempt to generate coherent emission of wavelength $118 \mathrm{~nm}$ and $71 \mathrm{~nm}$ which are third harmonic and the fifth of $355 \mathrm{~nm}$, respectively, thus we take simulation investigation for CHG on NIJI-VI storage ring, expected to direct experimental study.

In this paper, we mainly discussed three kinds of experimental designs to generate the third coherent harmonic wave, and analyzed the feasibility of these methods to find the optimal experimental design, and simulated the phase bunch, density modulation of electron beam and their revolution.

\section{Calculation Method}

When the pulse of pumping laser and the electron beam pass into the first undulator synchronously, the optical field of pumping laser will modulate the energy of electron beam. After bunched by dispersive section, the electron beam will generate coherent harmonic wave *Revised by H.L. Xu, NSRL,USTC, June 14, 2001

†hlxu@ustc.edu.cn radiation in the second undulator. The analytical calculation of the radiation emitted by an optical klystron is generally made with above simplifications ${ }^{[5]}$. In practical operation, energy modulation, beam bunched and radiation, the three proceed can not be distinguished, and these proceeds can also produce only in one undulator $^{[7]}$, not necessary for distinguishable optical klystron. So we must take numerical simulations of coherent harmonic generation.

Because the emittance of the beam in NIJI-VI storage ring is very small, the emittance will not be taken into account in follow simulation. And the simulation can be simplified by three assumptions:

The simulation can be simplified by three assumptions:

- It assumes also that optical wave of the pumping laser is plane-wave.

- Space charge effects may be ignored since the electron beam operates in the extreme-relativistic regime.

- Electron trajectories are not affected by the emitted radiation fields since the electron beam loses only a small fraction of its energy via undulator coherent harmonic emission.

The statistical averaging procedure is used in simulation. And the electron beam with Gauss distribution is divided into many equal segments which range is one wavelength of pumping laser, then each segment is placed on $n$ electrons with uniform distribution and the distribution of initial phase is $-\pi / 2 \leq \psi_{i 0} \leq \pi / 2$.This procedure ensures that unintended pre-bunching effects do not show up in the simulation results.

The calculation is based on the analytical expression of the trajectory of the electron in a sinusoidal magnetic field, given by the first Lorentz equation:

$$
\frac{d \vec{\beta}}{d t}=\frac{e}{m m} \vec{\beta} \times \vec{B}
$$

We did not take into account the possible imperfections of the magnetic field $\vec{B}$. We neglect also the effects of the electric field of pumping laser on this motion. Then

$$
\begin{aligned}
& \beta_{x}=\beta_{x 0}+\frac{K}{\gamma} \sin \left(\frac{2 \pi z}{\lambda_{u}}\right) \\
& \beta_{y}=\beta_{y 0} \\
& \beta_{z}=\sqrt{1-1 / \gamma^{2}-\beta_{x}^{2}-\beta_{y}^{2}}
\end{aligned}
$$


From this point, the energy of each electron is calculated all along the undulator, taking into account the external electric field, namely the second Lorentz equation:

$$
\frac{d \gamma}{d t}=\frac{e}{m c} \vec{E} \bullet \vec{\beta}
$$

The emitted electric field is computed from the Lienard-Wiechert potential equation:

$$
\begin{aligned}
& \frac{d I}{d \omega d \Omega}=\frac{e^{2} \omega^{2}}{16 \pi^{3} \varepsilon_{0} c^{3}} . \\
& \left|\int \vec{n} \times(\vec{n} \times \vec{\beta}) \exp \left(i\left(\omega t-\vec{k} \bullet \vec{z}-\Phi_{0}\right)\right) d z\right|^{2}
\end{aligned}
$$

where $\vec{n}$ is the direction of observation, and $\omega$ is the emitted optical frequency, $\Phi_{0}$ is the initial phase of electron radiation; so emitted power per electron was calculated.

At first, we compute the first undulator of $\mathrm{OK}$ (modulator). Then, the optical phase at the entrance of the second undulator (radiator) is newly computed for each particle taking into account its energy and the characteristics of dispersive section, we ensure the phase of entering dispersive section is $\Psi_{1}$ so the optical phase $\Psi_{2}$ at the entrance of radiator ${ }^{[8]}$ :

$$
\begin{aligned}
& \Psi_{2}=\Psi_{1}+2 \pi N_{d}\left(\lambda, \gamma_{R}\right)\left(\frac{\gamma_{R}}{\gamma_{i}}\right)^{2} \\
& +\frac{\omega L_{d} \theta_{i}^{2}}{2 c}+\frac{\omega}{c} \Delta x \theta_{x}^{i}
\end{aligned}
$$

where $L_{d}, \theta_{i}$ and $\Delta x$ are the length of dispersive section, incident angle of electron, and transverse position of electron, respectively . A passage into the radiator, with the new initial parameters for each particle, completes the calculation.

\section{Energy Modulation, Beam Bunched and Radiation Spectra}

The electron beam energy, which are modulated in different external laser fields, was calculated on entrance of the dispersive section.

The calculation results show that the bigger is the energy modulation the bigger is the power of the external laser. On the exit of the dispersive section, the electron density distributions after bunched are shown in Fig1. In the Fig. 1 (c), the electron beam was bunched effectively, which energy modulation transferred into density modulation, and will generate coherent harmonic radiation, calculation results were shown in Fig. 2

\section{Optimization of OK parameters and external laser field}

The wavelength of pumping laser is equal to the resonance wavelength of the optical klystron spontaneous emission, which was called as fundamental wave pumping, with this pumping method, according to the parameters of pumping Laser and optical klystron, the intensity of coherent third harmonic radiation were calculated when the external laser field varies, and simulation results are shown in Fig.3(solid circle). In same time, the radiation intensity calculated also when the dispersive section Nd varies, shown in Fig.4(solid circle) .

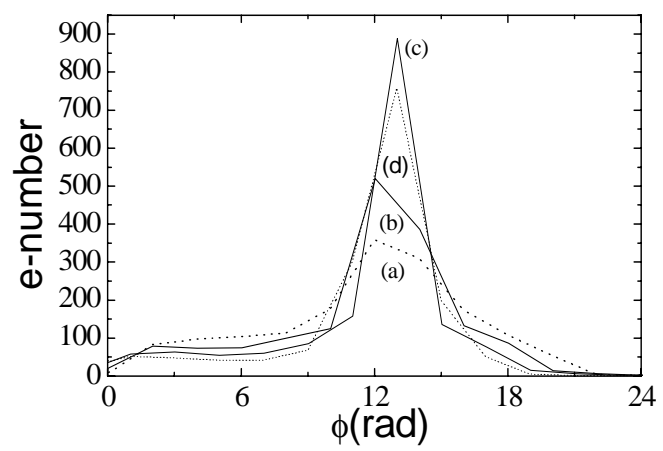

Fig.1 The density modulation $V S$ the electric field of pumping laser. $\mathrm{K}_{1}=\mathrm{K}_{2}=2.27, \mathrm{~N}_{\mathrm{d}}=5, \quad \gamma_{0}=604.7$ (a) $\mathrm{E}_{\mathrm{L}}=1.5$ $\times 10^{8} \mathrm{~V} / \mathrm{m}$, (b) $\mathrm{E}_{\mathrm{L}}=2.5 \times 10^{8} \mathrm{~V} / \mathrm{m}$, (c) $\mathrm{E}_{\mathrm{L}}=3.5 \times 10^{8} \mathrm{~V} / \mathrm{m}$, (d) $\mathrm{E}_{\mathrm{L}}=4.5 \times 10^{8} \mathrm{~V} / \mathrm{m}$

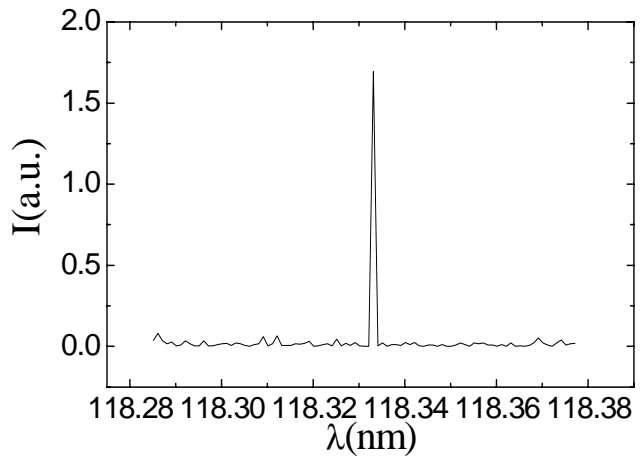

Fig.2 The spectrum of third coherent emission from the OK in NIJI-VI, $\mathrm{K}_{1}=\mathrm{K}_{2}=2.27, \mathrm{~N}_{\mathrm{d}}=5, \gamma_{0}=604.7, \mathrm{E}_{\mathrm{L}}=3.5 \mathrm{X}$ $10^{8} \mathrm{~V} / \mathrm{m}$

A relativistic electron in an undulator has a transverse velocity component given by formula (1) . Because energy is constant in the undulator, there is a longitudinal velocity oscillation at twice the transverse oscillation frequency. This produces "figure eight" motion when the electron orbit is viewed from a frame moving at the average forward velocity ${ }^{[1,4]}$ Viewed in the laboratory frame, there is a phase modulation of the emitted fundamental radiation, yielding the odd harmonic in the spectrum. This phase modulation also makes possible for harmonic pumping of $\mathrm{OK}$. The harmonic pumping method can also acquire coherent high harmonic radiation. To acquire the coherent third harmonic radiation of pumping wave which wavelength is $355 \mathrm{~nm}$ in NIJI-VI, the parameters of electron beam and $\mathrm{OK}$ will be changed, such as : $K=2.0$, and the electron energy must be damped to $\mathrm{E}=163.26 \mathrm{MeV}$. In this case, the intensity of coherent 
harmonic emission of optical klystron were calculated when both the external laser field and $\mathrm{Nd}$ vary, the numerical simulation results are showed in Fig.3 (solid line) and Fig.4 (solid line).

We take numeric simulation for the $\mathrm{CHG}$ of the non-symmetrical optical klystron configuration. In realty, only the magnet pole gap of the OK in NIJI-VI can be adjusted, namely the deflection parameter $\mathrm{K}$ can be adjusted, thus we make the second undulator resonant with the third harmonic of the fist undulator, and the numerical simulation results are shown in Fig.3(open circle) and Fig.4(open circle) with the method of fundamental pumping .

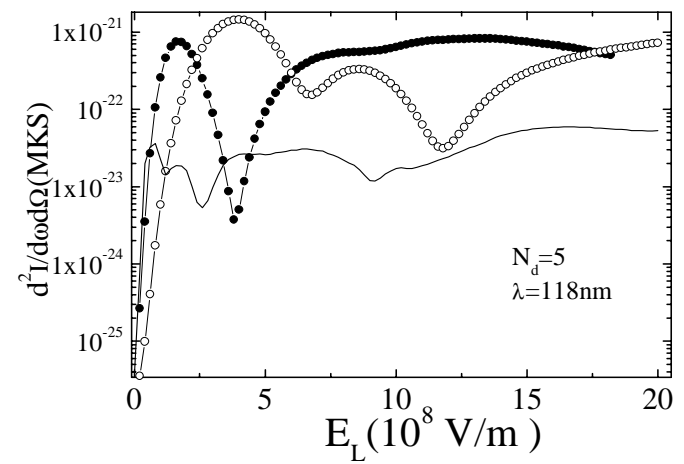

Fig.3 The intensity of coherent emission $V S$ the electric field of pumping laser in three kinds of designs

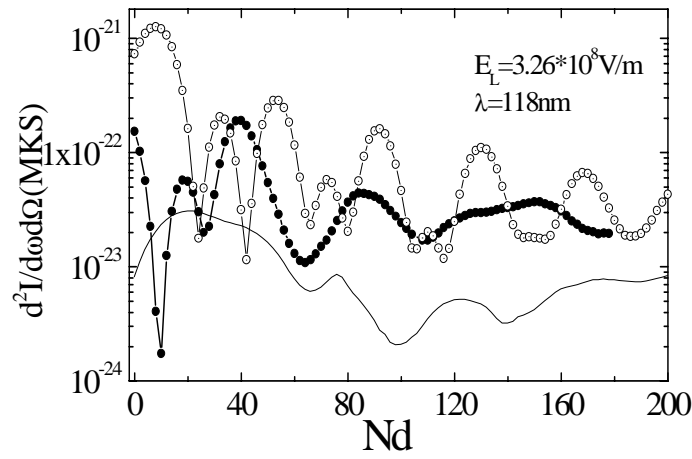

Fig.4 The intensity of coherent emission $V S \mathrm{Nd}$ of dispersive section in three kinds of designs

From Fig.3 and Fig.4, we found that the coherent emission intensity which the non-symmetrical optical klystron configuration emitted is little bigger than the intensity emitted by symmetrical optical klystron under the fundamental pumping, but when the coherent emission intensity become maximum it need the bigger power of pumping laser. The electric field of pumping laser $E_{L}$ is $3.26 \times 10^{8} \mathrm{~V} / \mathrm{m}$ by calculation according to the pumping laser parameters. With this value, the intensity of coherent emission VS the deflection parameter $\mathrm{K}^{2 \text { nd und }}$ were calculated, shown in Fig.5. From Fig.5 we find distinctly this conclusion, thus in our future $\mathrm{CHG}$ experiment we may use the method of non-symmetrical optical klystron and fundamental pumping.

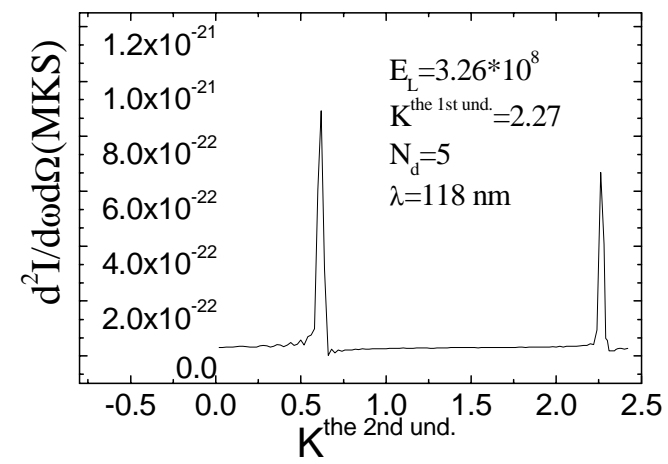

Fig 5 The intensity of coherent emission VS the deflection parameter $\mathrm{K}^{\text {2nd und }}$

When the parameter $\mathrm{Nd}$ of the dispersive section vary and $E_{L}$ keep constant which is equal to $3.26 \times 10^{8} \mathrm{~V} / \mathrm{m}$, we find that more smaller $\mathrm{Nd}$ is beneficial to improve the coherent emission intensity from Fig. 3 and Fig.4, thus $\mathrm{Nd}$ is better choice for $(0<\mathrm{Nd}<20)$.

\section{Conclusion}

From above analysis, the method of fundamental pumping is best choice for generating the coherent third harmonic emission to the CHG experiment in NIJI-VI , specifically if the optical klystron configuration is non-symmetrical, the coherent third harmonic emission can be improved distinctly. So, in the future $\mathrm{CHG}$ experiment the non-symmetrical optical klystron should be employed.

\section{Acknowledgments}

The work was performed under the auspices of the Quantum Radiation Division in ETL, supported by the Science and Technology Agency of Japan.

\section{References}

(1)G.N.Kulipanov,V.N.Litvinenko, .V.Pinaev,V.M.Popik, etal: NIM A296(1990)1.

(2)K.Yamada, N.Sei, T.Yamazaki, et al: Ipn. J.Appl.Phys. Vol.37(1998)pp.L1151-L1153

(3)P.L.Csonka, Particle Accl. Vol.8, pp.225-234, 1978

(4)P.Elleaume, J.pkys.(Paris), Vol. C1-44,pp.25-234

(5)R.Cosson, NIM,Vol.208, pp.185 -188,1983

(6)R.Prazeres, J.M.Ortega, C.Bazin,etal: Europhys.Lett. 4(1987)817

(7)S Werin, MEriksson: NIM A304 (1991)81-85

(8)P.Elleaume, Physics of Quantum Electronics, Vol.8, chapter 5, Addison-Wesley Publishig Company 